\title{
Ein Blick zurück in die Gegenwart
}

\author{
Marius Goldhorns Park im Spiegel des technischen Fortschritts
}

\section{Einleitung - Heine, Rilke und Goldhorn in Paris}

In den Jahren 1843, 1903 und 2019 schreibt jeweils ein deutscher Autor über die Stadt Paris. Heinrich Heine berichtet seine eigenen Eindrücke, Rainer Maria Rilkes Alter Ego Malte Laurids Brigge führt Tagebuch in der Stadt und Marius Goldhorn lässt seinen Protagonisten Arnold im Roman Park (2020) drei Tage in sommerlicher Hitze durch die Metropole taumeln. Sie alle erleben die Großstadt auch im Kontext der technischen Entwicklungen ihrer jeweiligen Epoche und mit einem dementsprechend unterschiedlichen Gefühl für Zeit und Raum. Auch wenn es sich um teilweise sehr verschiedene Texte handelt - nur ein Teil von Park spielt in Paris und Heines Berichte sind nicht fiktional - lohnt es sich zu Anfang zunächst alle drei in den Blick zu nehmen, bevor der Fokus auf Goldhorns Roman liegen wird. Was sie verbindet ist eine Wahrnehmung von Raum und Zeit im Angesicht technischen Fortschritts, der auf dieses Verhältnis entscheidenden Einfluss nimmt.

Als Heinrich Heine Mitte des 19. Jahrhunderts in Paris lebte und „Berichte über Politik, Kunst und Volksleben“ für die Augsburger Allgemeine Zeitung verfasste, die er 1852 in dem Band Lutetia zusammenstellte, schrieb er im Mai 1843 auch über ,[d]ie Eröffnung der beiden neuen Eisenbahnen, wovon die eine nach Orléans, die andere nach Rouen führt", ${ }^{1}$ und stellte - offenbar selbst bewegt von der Bedeutung der technischen Errungenschaft - eine „Erschütterung [fest], die jeder mitempfindet, wenn er nicht etwa auf einem socialen Isolirschemel steht“. ${ }^{2}$ Für Heine, der in einer Zeit aufgewachsen war, in der der Transport durch Pferdekraft über Land die einzige Beschleunigung gegenüber dem Laufen darstellte, muss die Entwicklung einer dampfbetriebenen Eisenbahn, die bereits Anfang der 1840er Jahre Geschwindigkeiten von bis zu 100 km/h erreichte, ähnlich gewirkt haben, wie für die Menschen des 21. Jahrhunderts das mobile Internet. „Mir ist, als kämen die Berge und Wälder aller Länder auf Paris angerückt. Ich rieche schon den Duft der deutschen Linden; vor meiner Thüre brandet die

1 Heinrich Heine: Lutezia. Berichte über Politik, Kunst und Volksleben. In: Heinrich Heine Säkularausgabe. Werke, Briefe, Lebenszeugnisse. Bearb. v. Lucienne Netter. Bd. 11. Berlin/Paris 1974, S. 180.

2 Ebd.

Ә Open Access. (c) 2022 Simon Sahner, publiziert von De Gruyter. (c) BY-NC-ND Dieses Werk ist lizenziert unter einer Creative Commons Namensnennung - Nicht-kommerziell - Keine Bearbeitung 4.0 International Lizenz. https://doi.org/10.1515/9783110758603-009 
Nordsee“, ${ }^{3}$ notiert Heine am 5. Mai 1843 in seinem Bericht für die Allgemeine Zeitung. Die Erfindung der Eisenbahn und die Möglichkeit Distanzen, deren Überbrückung ehemals mehrere Tagesreisen erforderte, in wenigen Stunden zu überwinden, veränderten die Wahrnehmung davon, wie Raum und Zeit zusammenhängen. Die Berge und Wälder, die mehrere hundert Kilometer entfernte Nordsee, sie scheinen für Heine näher gerückt zu sein, der Raum, in dem sich alles befindet, scheint sich verkleinert zu haben, weil sich sein Verhältnis zu Zeit verändert hat. ${ }^{4}$ Wolfgang Schivelbusch spricht in diesem Zusammenhang auch mit Blick auf die Textstelle bei Heine von einem Topos der „Vernichtung von Raum und Zeit“, mit „dem das frühe 19. Jahrhundert die Wirkung der Eisenbahn beschreibt“. 5

Etwa sechzig Jahre nach Heine schreibt Rainer Maria Rilke in Paris das diarische Prosabuch Die Aufzeichnungen des Malte Laurids Brigge. Inzwischen hatte sich die Einwohnerzahl der Stadt verdreifacht, sie hatte die Weltausstellung 1900 erlebt, war elektrifiziert worden und verfügte mit der Métro über ein U-Bahn-System. Die Geschwindigkeit und die Intensität, mit der Menschen, Waren und Informationen transportiert wurden und sich bewegten, hatte sich weiter erhöht. Raum und Zeit hatten ihr Verhältnis weiter verändert und die Welt war noch näher zusammengerückt. ${ }^{6}$ Der Tagebuchschreiber Brigge bemerkt: „Ich habe heute einen Brief geschrieben, dabei ist es mir aufgefallen, daß ich erst drei Wochen hier bin. Drei Wochen anderswo, auf dem Lande zum Beispiel, das konnte sein, wie ein Tag, hier sind es Jahre. “7 Was Brigge an dieser Stelle zu bemerken meint, ist eine Ausdehnung von Gegenwart, drei Wochen, ein relativ kurzer Zeitraum, fühlen sich an wie Jahre. Die Dichte des Geschehens um ihn herum, die Schnelligkeit des Austauschs von Menschen und Informationen dehnt das Zeiterleben und verkleinert den Raum. Gerade die großen Städte in der zweiten Hälfte des 19. und zu Beginn des 20. Jahrhunderts werden durch ihre Komplexität und technologischen Fortschritt zu etwas, das Armin Nassehi als „Synchronisationsmaschine“ bezeichnet. $^{8}$ Mit Blick auf die Stadt als Raum stellt Nassehi fest, dass hier „die Gleichzeitigkeit von Verschiedenem an einem

3 Ebd., S. 182.

4 Vgl. Wolfgang Schivelbusch: Geschichte der Eisenbahnreise. Zur Industrialisierung von Raum und Zeit im 19. Jahrhundert. Frankfurt a.M. 2018, S. $38 \mathrm{f}$.

5 Ebd., S. 35.

6 Vgl. Schivelbusch: Geschichte der Eisenbahnreise, S. 43.

7 Rainer Maria Rilke: Die Aufzeichnungen des Malte Laurids Brigge. Frankfurt a.M./Leipzig 1996, S. 11.

8 Vgl. Armin Nassehi: „Dichte Räume. Städte als Synchronisations- und Inklusionsmaschinen“. In: Martina Löw (Hg.): Differenzierungen des Städtischen. Opladen 2002, S. 211-232. 
Ort sichtbar wird“. ${ }^{9}$ Durch die Wahrnehmung von disparaten Geschehnissen zur gleichen Zeit am gleichen Ort kommt es zu einer „Dynamik der Zeit, der Beschleunigung und ungleicher Zeitreihen, die ständig koordiniert werden müssen. Städte synchronisieren unterschiedliche Zeitreihen von Verschiedenem. “10 Was sich bei Heine und Rilke in ihrer Wahrnehmung der großstädtischen Lebensweise andeutet und später durch die Erfindung des Telefons, des Flugzeugs und des Fernsehens fortsetzt, erreicht zu Beginn des 21. Jahrhunderts durch ein Netz aus digitalen Medien und schnellen Fortbewegungsmitteln seine aktuelle Verfassung: Die permanente Gleichzeitigkeit des Verschiedenen. Bereits im Jahr 1995, zu einer Zeit als ein eigener Internetzugang noch nicht den Standard darstellte und mobiles Internet noch ein Traum der Zukunft war, stellte Götz Großklaus fest: Die „alte Raum-Karte [wird] in den schnellen Gesellschaften unseres elektronischen Zeitalters abgelöst [...] durch eine neue ZeitKarte“. ${ }^{11}$ Diese Beobachtung einer Paradigmenverschiebung manifestiert sich schließlich in Marius Goldhorns Park als literarisches Abtasten von scheinbar endloser Gegenwart. ${ }^{12}$

Raum, Zeit und Medien hängen zusammen. Das ist keine neue Erkenntnis, aber es lohnt, sie sich noch einmal ins Gedächtnis zu rufen, wenn im Folgenden diese Zusammenhänge mit Blick auf Goldhorns Roman betrachtet werden sollen. Im Sinne der Medientheorie von Marshall McLuhan und der Annahme, dass Medien im weitesten Sinne Transportmittel sind, ist bereits die Eisenbahn bei Heine ein Medium. ${ }^{13}$ Sie leistet als solches ,die Übertragung von etwas (das noch genauer zu bestimmen wäre) von einem Punkt in Raum und Zeit zu einem anderen (der noch genauer zu bestimmen wäre)“. ${ }^{14}$ Mit der Erfindung der

9 Ebd., S. 215.

10 Ebd.

11 Götz Großklaus: Medien-Zeit, Raum-Zeit. Zum Wandel der raumzeitlichen Wahrnehmung in der Moderne. Frankfurt a.M. 1995, S. 103.

12 Vgl. Endlose Gegenwart. Donaufestival-Reader Vol. 2. Krems 2018.

13 Schivelbusch stellt gar eine konkrete Verbindung zwischen dem Computer und der Eisenbahn her, die beide Versuche seien, „die Welt über die von ihnen erzeugten Erscheinungen nachzubilden und zu reproduzieren. Beide sind erfolgreich. Und sie erreichen ihr Ziel durch ihre Machinationen. Ob die Welt, die sie erschaffen, das globale Netzwerk einer dampfgetriebenen industriellen Produktion und Beförderung ist oder die digitalisierte Cyberwelt der Information - es ist ihre Weltmaschine.“ (Wolfgang Schivelbusch: „Weltmaschinen: Die Dampfmaschine, die Eisenbahn und der Computer. Vorwort zur amerikanischen Neuausgabe 2014“. In: Ders.: Geschichte der Eisenbahnreise. Zur Industrialisierung von Raum und Zeit im 19. Jahrhundert. Frankfurt a.M. 2018, S. I-X, hier S. IV).

14 Lorenz Engell: „Wege, Kanäle, Übertragungen. Zur Einführung.“ In: Claus Pias u. a. (Hg.): Kursbuch Medienkultur. Die maßgeblichen Theorien von Brecht bis Baudrillard. Düsseldorf 1999, S. 127-133, hier S. 127. 
Dampfmaschine und bald darauf der Eisenbahn kommt es zu einer Steigerung von etwas, das Großklaus als „Grad der zeit-räumlichen Durchstrukturierung “'15 bezeichnet. Je höher dieser Grad sei, desto höher sei - mit Norbert Elias gesprochen - auch „der Grad ,der kognitiven Meisterung von Zusammenhängen in Raum und Zeit'“. ${ }^{16}$ Das führe dazu, dass jede Generation von Medien ${ }^{17}$,jeweils die gültigen Parameter von Raum und Zeit, von raumzeitlicher Nähe und Ferne revolutioniert und neue Zusammenhänge von Raum und Zeit“18 stiftet. Heine, Rilke und Goldhorn verarbeiten diese jeweils neuen Zusammenhänge im medialen und technischen Kontext ihrer Zeit und stellen dadurch literarisch eine sich immer weiter verdichtende Gegenwart dar, die im digitalen Nomadentum von Arnold in Park ihren vorläufigen Höhepunkt erfährt. Soweit die These der folgenden textnahen Betrachtung des Romans im Kontext aktueller zeittheoretischer Gegenwartsanalysen.

\section{Reisen auf Zeit-Karten - Gegenwart in Park}

Über hundert Jahre nach Brigge reist Arnold, der Protagonist des kurzen Romans Park von Marius Goldhorn, mit dem Zug von Berlin nach Paris, um von dort drei Tage später nach Athen weiterzufliegen, wo er seiner Ex-Freundin Odile bei einem Filmdreh helfen soll. In seinem Erleben der Stadt, das nur wenige Tage währt, ist die Gegenwart noch weiter ausgedehnt, immer wieder geht sein Blick auf die Zeitanzeige seines Smartphone, bereits der zweite Satz des Romans markiert die Zeit: „Es war 14.21 Uhr.“" ${ }^{\text {"19 }}$ Während der Zugfahrt schreibt er in einem Chat mit seinem Freund Veysel in Berlin, verschickt Nachrichten an Odile in Athen, die unbeantwortet bleiben, liest alte Nachrichten von ihr, löscht sie und kommt „[e]ine Stunde und sechsundzwanzig Minuten später“20 in Paris an. Fast wie eine Reminiszenz an seine literarischen Vorgänger wirkt da seine Vorstellung „wie Odile in einer Bahnhofshalle aus dem 19. Jahrhundert, an eine

15 Großklaus: Medien-Zeit, Raum-Zeit, S. 12.

16 Ebd.

17 Es sei angemerkt, dass Großklaus eine kategorische Unterscheidung zwischen „Generation von Bewegungsmaschinen“ (Eisenbahn, Auto, Flugzeug etc.) und „neue Mediengenerationen“ (Fotografie, Film, Computer etc.) vornimmt. Im Sinne eines erweiterten Medienbegriffs kann aber für alle diese Fälle von Medien die Rede sein.

18 Großklaus: Medien-Zeit, Raum-Zeit, S. 12.

19 Marius Goldhorn: Park. Frankfurt a.M. ${ }^{2} 2020$, S. 9.

20 Ebd., S. 14. 
gusseiserne Säule gelehnt, auf ihn wartete“. ${ }^{21}$ Anders als bei Heine und Rilke sind hier Eisenbahn und die Métro aber nur der geringste Teil eines medialen Zusammenhangs, der das Gegenwartserleben von Arnold beinahe bis zur temporalen Erstarrung treibt.

Übernimmt man den Begriff der,Zeit-Karte، von Großklaus, dann lässt sich mit Blick auf die Fortbewegung von Arnold feststellen, dass im Zentrum seines Reisens tatsächlich Zeit als entscheidende Kategorie steht. Zwar bewegt er sich innerhalb von wenigen Tagen von Berlin nach Paris und dann weiter nach Athen, die europäischen Metropolen sind jedoch nur der Raum, in dem sich Gegenwart als Zeit konstituiert. Entscheidend ist nicht der Ort, sondern die Zeit. Arnold ist ständig eingebunden in eine Markierung von Gegenwart. Auf beinahe penetrante Weise hält er die Zeit fest und positioniert sich in einem Gefüge von Gegenwart, Zukunft und Vergangenheit. Dabei ist Arnolds Erleben zunächst vor allem geprägt von einem Verschränken und Verschieben von Zeitebenen. Bereits auf dem Weg nach Paris, das selbst nur Durchgangsstation ist, fallen mehrere Zeiten zusammen. Das Lesen und darauffolgende Löschen der Nachrichten von Odile, die vor allem aus der Zeit ihrer ersten Begegnung und ihrer kurzen Beziehung stammen, holen die Vergangenheit zunächst in die Gegenwart. Sie ist nun nicht mehr in Gänze nachvollziehbar, sondern rückt an die Gegenwart heran; bis zu dem Punkt als Odile wenige Wochen vorher wieder Kontakt aufgenommen hat. Sobald er in Paris angekommen ist, setzt er sich wieder in Bezug zu einer Zeit-Karte, indem er feststellt: „Es war 17.03 Uhr. Er dachte: In drei Tagen bin ich in Athen.“22 Durch den sich immer wiederholenden Blick auf das Smartphone und das Festhalten der aktuellen Uhrzeit, wird eine permanente Gegenwart konstituiert. Jedes Erleben ist immer im Hier und Jetzt. Das zeigt sich auch im wiederholten Aktualisieren von E-Mails und Nachrichten, das häufig mit dem Blick auf die Uhrzeitanzeige auf dem Display einhergeht. Durch das Abrufen der aktuellen Nachrichten oder zumindest durch die Versicherung, dass man keine Nachrichten verpasst hat, wird ein Gegenwärtigbleiben inszeniert, das nicht zulässt, dass eine Diskrepanz zwischen der Wahrnehmung und dem Verlauf der Zeit entsteht.

Was Arnold zunächst vor allem in den Tagen in Paris erlebt, was sich aber als thematisches und strukturelles Zentrum durch die gesamte Erzählung zieht, ist das Gefühl einer permanenten Gegenwart, in der sich Zeit auf engstem Raum verdichtet. Damit schließt der Roman erkennbar an aktuelle Zeit-Diskurse zur Wahrnehmung von Gegenwart im Kontext digitaler Medien an. In seinem Buch

21 Ebd.

22 Ebd. 
Present Shock: When Everything Happens Now (2013) macht Douglas Rushkoff dieses Zeiterleben zum Fokus seiner Betrachtung der Gegenwart. Seiner These zufolge hat die digitalisierte Gesellschaft ihre Wahrnehmung und ihr Denken, kurz ihr ganzes Sein auf ein immerwährendes Jetzt projiziert. Während das Ende des 20. Jahrhunderts von einem Futurism, einer Fokussierung auf das Zukünftige, gekennzeichnet gewesen sei, sei der Beginn des nächsten Jahrhunderts geprägt von Presentism, einer starren Perspektive auf die Gegenwart. ${ }^{23}$ Das sei nicht zuletzt die Folge fehlender, großer Erzählungen, die eine klare temporale Struktur vorgeben und in eine Zukunft weisen könnten: „Our digital devices and the outlooks they inspired allowed us to break free of the often repressive timelines of our storytellers, turning us from creatures led about by future expectations into more fully present-oriented human beings. “24 Dabei ist diese Orientierung an einer Gegenwart laut Rushkoff nicht in dem Maße erstrebenswert, wie sie in dem Zitat zunächst scheinen mag. Vielmehr erkennt er in dem festgestellten Drang, auf digitalen Endgeräten Ereignisse an unterschiedlichen Orten auf der ganzen Welt gleichzeitig zu verfolgen, eine Dissoziation des Gegenwartserlebens. In dem unermüdlichen Bestreben gegenwärtig zu sein, verlieren wir - so Rushkoff - die Gegenwart in Wahrheit aus dem Blick:

By dividing our attention between our digital extensions, we sacrifice our connection to the truer present in which we are living. The tension between the faux present of digital bombardment and the true now of a coherently living human generates the second kind of present shock, what we're calling digiphrenia - digi for ,digital, ' and phrenia for ,dissordered condition of mental activity. ${ }^{25}$

In Arnolds Verhalten äußert sich das, was Rushkoff Digiphrenia nennt: Eine psychische Spannung, die daraus entsteht, dass das konkrete Erleben einer lokalen Gegenwärtigkeit, dem sprichwörtlichen Hier-und-Jetzt-Sein, in Konflikt gerät mit einem Zeiterleben im digitalen Raum, dessen Ziel zum einen ist, nicht hinter das aktuelle Geschehen zurückzufallen, gegenwärtig zu sein, zum anderen aber auch die Möglichkeit bietet, unterschiedliche Zeiten in den Raum der Gegenwart zu holen. Für Arnold resultiert daraus ein Überfliegen von Handlungen, visuellen Eindrücken und Informationen, die aber nicht verarbeitet werden:

Er dachte: Eigentlich ist alles in Ordnung im Moment. Er schaltete den Fernseher ein. Ein Kleinkind trug einen viel zu großen Hirnstrommesser mit roten und blauen Elektroden auf dem Kopf. Arnold wurde sehr müde. Arnold wachte auf. Es war 11.02 Uhr. Arnold

23 Vgl. Douglas Rushkoff: Present Shock: When Everything Happens Now. New York 2013, S. 3. Siehe auch den Beitrag von Eckhard Schumacher in diesem Band.

24 Rushkoff: Present Shock, S. 72 f.

25 Ebd., S. 75. 
hatte neun Stunden und dreizehn Minuten geschlafen. Arnold setzte sich auf. Er dachte: Morgen fliege ich nach Athen. Er dachte an Odile. Er suchte nach einem frischen T-Shirt. Arnold nahm sein MacBook und las das Gedicht über die Aliens. Arnold dachte: Wie komme ich darauf, dass sie die Erde zerstören wollen? Vielleicht wollten sie mich nur über ihre Ankunft informieren. Er machte Acid Mt. Fuji von Susumu Yokota an. Er schaute nach seinem Kontostand auf der Website seiner Bank. Er war okay. Arnold las den WikipediaEintrag der Terra-Nova-Expedition. Er dachte darüber nach zu masturbieren. Arnold fragte sich, ob er vollkommen verzweifelt oder eigentlich alles in Ordnung war. ${ }^{26}$

Die Frage, die sich Arnold am Ende dieses Abschnitts stellt, soll wohl eine Dissonanz zwischen dem eigenen Erleben und der Wahrnehmung disparater Eindrücke zur gleichen Zeit ausdrücken. Die Voraussetzung für diese Empfindung ist die Annahme, dass die sinnliche Wahrnehmung des Raumes, das physische Sein im Hier-und-Jetzt, in Konflikt gerät mit einem vor allem medial vermittelten Gegenwartsgeschehen. Großklaus zufolge hat in dieser Medienrealität „nichts ,seinen Ort', sondern alles ,seine Zeit“““, 27 dem gegenüber steht jedoch der Mensch als Körper, der immer seinen Ort haben muss: „All experience is local. Everything we see, hear, touch, smell, and taste is experienced through our bodies. And unless one believes in out-of-body experiences, one accepts that we and our bodies are permanently fused. We are always in place, and place is always with us.“28 Diese Setzung einer Körpererfahrung, die zwingend einen Ort haben muss, steht zu Beginn des Textes „The Rise of Glocality. New Senses of Place and Identiy in the Global Village” von Joshua Meyrowitz. In seinem Beitrag aus dem Jahr 2005 formuliert Meyrowitz mit Blick auf den damals aktuellen Stand der digitalen Technik und des Internets seine Interpretation des Begriffs Glocality als das Eingebundensein in eine ,interconnected global matrix “, ${ }^{29}$ daher existieren das Lokale und das Globale gleichzeitig im Status der Glokalität. ${ }^{30}$ Dieses Leben in Glokalitäten führe jedoch zu einer „dissociation between physical place and experiantial space“.31

Was in Arnolds Erleben in Park und bei der Diagnose eines Present Shock durch Rushkoff direkt zusammenhängt mit der Möglichkeit digitaler Kommunikation und dem permanenten Zugriff auf Nachrichten und Informationen im Internet - das, was Hans Ulrich Gumbrecht an anderer Stelle als „Hyperkom-

26 Goldhorn: Park, S. 36f.

27 Großklaus: Medien-Zeit, Raum-Zeit, S. 112.

28 Joshua Meyrowitz: „The Rise of Glocality. New Senses of Place and Identity in the Global Village“. In: Kristóf Nyíri (Hg.): A Sense of Place: The Global and the Local in Mobile Communication. Wien 2005, 21-30, hier S. 21.

29 Ebd., S. 23.

30 Vgl. ebd. S. 25.

31 Ebd., S. 27. 
munikation“ beschrieben hat ${ }^{32}$ - konstatierte also Großklaus bereits $1995^{33}$ und Meyrowitz gar schon 1987. In seiner Monografie Die Fernseh-Gesellschaft stellt Meyrowitz bereits Ende der achtziger Jahre, zu einer Zeit als das Internet in der heutigen Form noch nicht existierte, fest: „Die elektronischen Medien haben die Bedeutung von Ort, Zeit und physischen Barrieren als Einflußgrößen der Kommunikation nachhaltig verändert.“34 Den Grund dafür findet er bereits damals in der Möglichkeit, Gegenwartsgeschehen wahrzunehmen, das sich nicht am gleichen Ort vollzieht, an dem sich der wahrnehmende Mensch befindet. ${ }^{35}$ Das Gefühl einer Dissoziation angesichts zeit-räumlicher Verschiebungen aufgrund technischen Fortschritts ist demnach nichts, was sich erst im Zeitalter weit verbreiteter digitaler Medien und eines mobilen Internetzuganges zeigt, sondern von Großklaus, Meyrowitz und anderen bereits mit Blick auf das Fernsehen gedacht wurde. Sogar Heines Gefühl eines kleiner gewordenen Raumes mittels einer schnelleren Mobilität - die Nordsee scheint ihm bereits vor seiner Pariser Haustür zu beginnen - ist in gewisser Weise bereits eine ,Digiphrenie‘ avant la lettre. ${ }^{36}$ Die Landschaften, die im 19. Jahrhundert aufgrund der neuen, schnellen Reise- und Kommunikationsmöglichkeiten, „ihr Jetzt in einem ganz konkreten Sinne verlieren“, ${ }^{37}$ so Schivelbusch, sind daher bereits Vorboten einer Wahrnehmungsveränderung durch elektronische Medien, die Arnold erfährt. Die These eines Present Shock, der Rushkoff zufolge das neue Jahrtausend aufgrund der sich weiter verdichtenden Gegenwartswahrnehmung durch fortgeschrittene Digitaltechnik prägt, operiert daher mit Prämissen, die sich bereits zu Zeiten finden lassen, die nach Rushkoff noch von einem Zukunftsdenken bestimmt waren und teilweise Digitalität im heutigen Sinne noch gar nicht kannten. Das falsifiziert Rushkoffs Diagnose zwar nicht, implementiert sie jedoch in einen größeren Zusammenhang von Zeit-Raum-Verhältnissen, die an technische Entwicklungen gebunden sind. Damit erscheint die Fokussierung auf Gegenwart, die Rushkoff ausmacht, nicht mehr als Zäsur, sondern vielmehr als Ergebnis einer forcierten Entwicklung, die bereits im 19. Jahrhundert begonnen hat.

32 Vgl. Hans Ulrich Gumbrecht: Unsere breite Gegenwart. Berlin 2010, S. 114-131.

33 Vgl. Großklaus: Medien-Zeit, Raum-Zeit, S. 108-112.

34 Joshua Meyrowitz: Die Fernseh-Gesellschaft. Wirklichkeit und Identität im Medienzeitalter. Weinheim/Basel 1987, S. 21.

35 Vgl. ebd.

36 Mit Blick auf die These von Armin Nassehi, dass bereits die Gesellschaften des 19. Jahrhunderts strukturell digital organisiert gewesen seien, ließe sich gar die Frage stellen, ob der von Rushkoff eingeführte Begriff der Digiphrenia an dieser Stelle nicht sogar besser passt, als man auf den ersten Blick vermuten würde. (Siehe dazu: Armin Nassehi: Muster. Theorie der digitalen Gesellschaft. München 2019, insbesondere S. 57-63.)

37 Schivelbusch: Die Geschichte der Eisenbahnreise, S. 43. 


\section{Erzeugung von Vergangenheit und Zukunft durch Semantisierung}

In Park manifestiert sich insbesondere im zweiten Teil des Romans, der in der nahen Vergangenheit spielt, wie sehr diese Orientierung an einer Zeit-Karte mit digitaler Technik zusammengedacht wird. Die Fixierung auf Uhrzeiten, die Anzeige und Markierung von Zeiten und Zeitabläufen, zum Beispiel dem minutengenauen Festhalten der Schlafenszeit, ist im Vergleich zu den Tagen in Paris sogar gesteigert:

Vier Tage später stand Arnold in seiner Küche. Er rührte ein Vitaminpulver in ein Glas Leitungswasser. Er blickte auf die Waschmittelreste in der offen stehenden Waschmaschinenschublade. Er entsperrte sein iPhone. Es war 12.12 Uhr. Arnold dachte: 12.12 Uhr. Er dachte: Mir kommt es vor, als sei alles um mich herum gemacht. Arnold schaute aus dem Fenster. Er betrachtete die Bäume im Sommerwind. Arnold dachte: Das ist viel zu schön. Arnold blickte auf sein iPhone. Es war 12.14 Uhr. Er betrachtete die Waschmaschine. Arnold versuchte vierzig Minuten lang, ein Gedicht zu schreiben. ${ }^{38}$

Die genaue Taktung von Handlungsabläufen, die entlang einer Zeitlinie verlaufen, die Arnold durch das häufige Festhalten der Uhrzeit auf seinem iPhone erst entstehen lässt, dehnt die Wahrnehmung dieser Abläufe im Verhältnis zur Zeit aus. Dadurch, dass jede Handlung in eine Beziehung zu der Zeit gestellt wird, innerhalb der sie abläuft, verliert die einzelne Handlung an Bedeutung. Sie steht lediglich noch für eine Position auf einem Zeitstrahl. Parallel dazu scheint die Zeit gedehnt zu werden. Besonders deutlich wird das am Zusammenhang des obigen Zitats mit einem kurz darauffolgenden Ausschnitt:

Zwei Tage später war es Samstag. Arnold lag auf dem Boden und stellte sich eine Leiche auf einer Matratze mit Memory-Schaum vor. Er entsperrte sein iPhone. Es war 12.12 Uhr. Arnold dachte: 12.12 Uhr. Ist das Zufall? Oder sehe ich so oft diese Uhrzeit, weil ich so oft auf die Uhr schaue? Intensiviere ich die Wahrscheinlichkeit dieses Phänomens? Erzwinge ich durch Wiederholung Mystik? Erhöhe ich die Erfahrung des Sinnvollen, wenn ich nur häufig genug das Gleiche tue ${ }^{39}$

Dieser Moment der temporalen Erstarrung einer Gegenwartserfahrung kann als zentrale Aussage des Romans gelesen werden. Die Wiederholbarkeit von Zeit und ihres Erlebens führen zum vollständigen Stillstand der Gegenwart. Die semantische Leere des eigenen Tuns und Wahrnehmens wird durch stetige Wiederholung zu umgehen versucht. Diese Wiederholung geht jedoch einher mit

38 Goldhorn: Park, S. $64 \mathrm{f}$.

39 Ebd., S. 66. 
einem Bewusstsein für Zeit, wodurch sich auch die Zeit stets zu wiederholen scheint. Das Zeiterleben von Arnold ist ein Phänomen, das auch Boris Groys beschreibt: „The present has ceased to be a point of transition from the past to the future, becoming instead a site of the permanent rewriting of both past and future [...]." ${ }^{40}$ Was Groys anhand von Gegenwartskunst erkennt, die sich als das benannte permanent rewriting darstellt, zeigt sich in Goldhorns Roman als die Repetition von bedeutungslosen Vorgängen, die den Eindruck einer zeitlosen Gegenwart konstruieren. Dieser Verlust eines Gefühls für ein Vorher und ein Danach führt zu etwas, das Groys als „phenomenon of unproductive wasted time ${ }^{\text {411 }}$ bezeichnet. Zeit wird registriert und markiert, sie wird jedoch nicht semantisch gefüllt, wodurch einerseits das Gefühl verschwendeter Zeit entsteht, andererseits aber der Eindruck einer endlosen Gegenwart. Die repetitiven und bedeutungslosen Handlungen markieren keine Punkte auf der Ebene der Zeit, zu denen sich Arnold in Beziehung setzen könnte, alles ist Gegenwart, weil es keine Vergangenheit und keine Zukunft gibt.

Das Kontrastfeld zu diesem totalen Stillstand bei gleichzeitiger Fokussierung auf Zeit, bildet zumindest für einige Wochen die Beziehung mit Odile, die im Verlauf der Handlungsvergangenheit auf die Phase der totalen Gegenwartserstarrung folgt. Ab dem Zeitpunkt, da Odile in Arnolds Leben tritt, erfährt er Momente, die mit Bedeutung aufgeladen sind. Als er am Morgen nach der ersten gemeinsamen Nacht die Wohnung verlässt, um einzukaufen, verbringt er „keine Sekunde zu lang im Kiosk“. ${ }^{42}$ Die Anwesenheit von Odile in seinem Leben gibt seinem Handeln instantan eine Bedeutung, als wäre mit Odile eine Zäsur in den Verlauf der Zeit eingefügt worden, die eine Vergangenheit sowie eine Zukunft markiert:

Odile sagte: Morgen kaufe ich Remoulade.

Arnold dachte: Morgen.

Odile sagte: Eigentlich finde ich deine Wohnung sehr schön.

Er sagte: Morgen kaufe ich eine große Tube Remoulade. ${ }^{43}$

Es scheint, als würde Arnold in diesem Moment selbst klar werden, dass es nun eine Zeitdimension gibt, die nicht mehr direkt erfahrbar ist, sondern die auf eine konkrete Zukunft verweist, auf ein Morgen. Die Zeit, die er gemeinsam mit Odile verbringt, wird von Arnold nicht mehr als starre Gegenwart wahrgenommen, sondern als ein sinnhaftes Vergehen von Zeit. Paradoxerweise führt eben

40 Boris Groys: „Comrades of Time“. In: e-flux journal 11(2009), unpaginiert.

41 Ebd.

42 Goldhorn: Park, S. 95.

43 Ebd., S. 97. 
diese Sinnhaftigkeit des Erlebten dazu, dass das Verhältnis von Zeit und Erleben als irrelevant wahrgenommen wird. Beinahe plakativ mutet an, wie Odile die Zeitwahrnehmung Arnolds aktiv außer Kraft setzt, als sie auf seine Frage nach der Uhrzeit antwortet mit „Keine Ahnung“. ${ }^{44}$ Hatte Arnold bis zu diesem Punkt der Erzählung seine Zeit durch den zwanghaften Blick auf sein Smartphone strukturiert und festgehalten, scheint er nun von diesem Diktat befreit zu sein.

Die Zeit der Beziehung, insgesamt sechs Monate, ist lediglich durch parataktische Ereignissätze beschrieben, die durch eine iterative Erzählweise den Verlauf eines gemeinsamen Erlebens schildern:

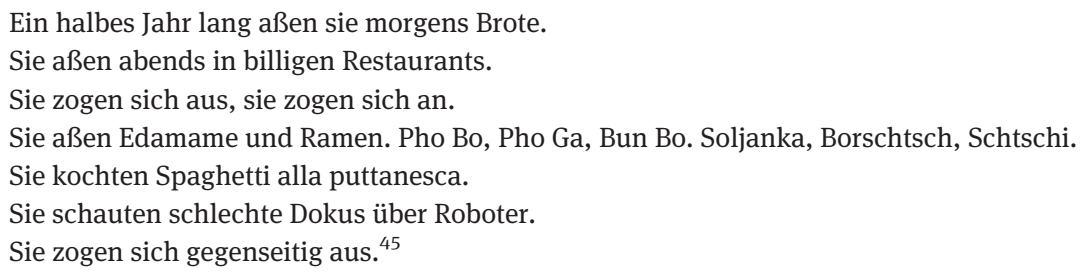

Insgesamt vierzig dieser gemeinsamen, teilweise sehr spezifischen Handlungen werden aufgelistet, bevor Odile Arnold verlässt, um nach London zu gehen. Virulent ist hier nicht nur das Verhältnis von erzählter Zeit zu Erzählzeit, das im Vergleich eine wesentlich stärkere Raffung aufweist, sondern insbesondere auch die weitgehende Abwesenheit von digitaler Technik und einer Zeiterfassung - zwei Elemente, die das Erleben Arnolds bis zu diesem Moment der Handlung geprägt hatten. Zeit wird in diesem Sinne als Orientierungsgröße bedeutungslos, sobald sie nur als Teil eines bedeutungsvollen Handelns wahrgenommen wird: Sechs Monate vergehen, ohne dass Arnold es bemerkt, könnte man es formulieren. Er erlebt Gegenwart, ohne sie als solche bewusst wahrzunehmen. Da die Diskrepanz zwischen einem lokalen und digitalen, globalen Gegenwartserleben drastisch verringert worden ist, verschwindet auch das Gefühl einer Dissoziation zwischen dem „physical place and experiential space“, ${ }^{46}$ um an dieser Stelle noch einmal Meyrowitz aufzurufen. Diese kurze Episode in der erzählten Vergangenheit des Romans stellt die einzige Phase der Erzählung dar, in der Zeit eine bewusst untergeordnete Rolle spielt.

44 Ebd., S. 99.

45 Ebd., S. 101.

46 Meyrowitz: „The Rise of Glocality“, S. 27. 


\section{Sehnsucht nach einem Vorher und einem Nachher}

Mit der Ankunft in Athen in der Erzählgegenwart beginnt ein Bewusstsein für Zeitempfinden einzusetzen, das sich von dem zwanghaften Gegenwartserleben in Form einer Erstarrung insofern unterscheidet, als Arnold sich nun in Bezug zu dieser Wahrnehmung setzt. Als er Athen erreicht und dort wieder auf Odile trifft, läge die Vermutung nahe, dass es zu einem Wechsel des Zeitempfindens kommt, der dem der ersten Phase der Beziehung ähnelt. Stattdessen markiert das Erreichen Athens und das Zusammentreffen mit Odile den Beginn einer anderen Wahrnehmung Arnolds von sich selbst im Kontext von Gegenwart. Dies geschieht jedoch weniger bewusst als vielmehr in Form einer sublimen Perzeption von temporalen Zusammenhängen, ohne dabei zunächst eine konkrete Reflexion über diese Veränderung zu initiieren.

Im Gespräch mit Odiles Freundin und Mitbewohnerin Esther erfährt er bereits kurz nach seiner Ankunft eine Unsicherheit der eigenen Wahrnehmung. Während der drei Tage in Paris hatte er einen terroristischen Anschlag in der Stadt in den Nachrichten verfolgt, kann sich aber nun - nur wenige Tage später an nichts Genaues mehr erinnern. Das nur kurze Zeit zurückliegende Ereignis verschwimmt bereits mit anderen Wahrnehmungen, die gleichzeitig stattgefunden haben: „Arnold wurde klar, dass er das tatsächlich nicht wusste. Er erinnerte sich nur vage an die Berichterstattung. Was hatte er gemacht? Über die Aliens nachgedacht? Arnold dachte an sein Gedicht. In ihm flackerten erschöpfte Bilder von Sand auf, ein Strand, eine Wüste. Er atmete tief durch. “47 Verstärkt wird diese Unsicherheit über die eigene Erinnerung noch durch Esthers Erzählungen von einem anderen, früheren Anschlag in Paris, als sie selbst einmal dort war, und dadurch, dass Odile nichts zu dem aktuellen Anschlag in den Nachrichten finden kann. Diese Vagheit, das Verschwimmen von Ereignissen, die sich nicht mehr einer Zeit ihres Geschehens zuordnen lassen, und Odiles finalisierende Feststellung, dass es „[e]gal“48 sei, sind eine zunächst unbewusste Irritation von Arnolds eigener Zeitwahrnehmung. Der Wunsch nach einer Katastrophe, „damit alles wieder an seinen Platz rückt“" ${ }^{49}$ ist als Folge davon zum ersten Mal die konkrete Suche nach etwas, das sich zuvor lediglich im Subtext seiner Zeitwahrnehmung erkennen ließ: Es bedarf eines semantisierten Einschnitts, der die permanente Gegenwart in ein Vorher und Nachher trennt. Arnolds Tagtraum davon, „wie

47 Goldhorn: Park, S. 113.

48 Ebd., S. 114.

49 Ebd., S. 115. 
Odile und er sich im Ascheregen küssten“, 50 ist nicht nur eine medial geprägte Idealvorstellung einer Vereinigung zweier Menschen, sondern auch die Sehnsucht nach einem Moment, der - hier in Form einer miterlebten Katastrophe - als bedeutungsgeladen erfahren wird.

Was Arnold in der Folge während der Zeit in Athen erfährt, könnte man als den Wunsch nach einer temporalen Tiefendimension beschreiben. Die Irritation über die Realität der eigenen Erinnerungen angesichts einer Gegenwart, die sich aus sich überlagernden Ereignissen auftürmt, entlädt sich in einer Sensibilität für Zeitebenen, die sich in Vergangenheit, Gegenwart und Zukunft einteilen lassen. Seine Idee für eine Funktion eines Textverarbeitungsprogramms, „mit der man einen ganzen Text aus dem Präsens ins Präteritum setzen könnte“, ${ }^{51}$ ist ebenso wie seine Vorstellung von Gebäuden der Gegenwart als Ruinen der Zukunft die bereits erwähnte sublime Perzeption von Zeitverhältnissen. Der Anblick der lediglich etwas mehr als fünfzehn Jahre alten Gebäude der olympischen Spiele von 2004 löst bei Arnold das plötzliche Gefühl einer existierenden Vergangenheit und einer bevorstehenden Zukunft aus:

Ihm kam das verfallene Areal edel vor, vielleicht, weil es ein noch relativ unberührtes Bruchstück war. Erinnerung und Gegenwart fielen ineinander, und in diesem Moment wurde ihm klar, was das für ein ungeheurer Fortschritt im menschlichen Denken war, das ästhetische Bewusstsein der Ruine, die Musealisierung der Welt. Hätte Odile Arnold nicht gefragt, ob er das Stativ aufbauen könne, er hätte sich für einen kurzen Moment im Einklang mit seiner Umwelt befunden. Er zog das Aluminium-Stativ auseinander. Er dachte: Alles, was der Mensch macht, ist, die Erde in eine Ruine zu verwandeln, einen eingezäunten Abgrund zu hinterlassen, aber für wen? Arnold sagte: Wie eine Ruine aus der Zukunft. ${ }^{52}$

Das Olympia-Areal, das nur wenige Jahre nach seiner Erbauung seinen Zweck bereits verloren $\mathrm{zu}$ haben scheint, ist ein Ort, an dem Vergangenheit, Gegenwart und Zukunft gleichzeitig zu existieren scheinen. Die im historischen Vergleich geringe Zeitspanne, in der die Gebäude erbaut, genutzt und ihrem Verfallen überlassen wurden, verdichtet eine Zeiterfahrung, die sich sonst über mehrere Dekaden oder gar Jahrhunderte erstreckt. Diese heterotopische Qualität des Ortes spürt Arnold zwar, eine aktive Reflexion über die Bedeutung und die Gründe dieser Empfindung bleibt jedoch aus.

Doch trotz dieser erhöhten Sensibilität für temporale Zusammenhänge ist Arnold nicht in der Lage die von ihm erhoffte Katastrophe, die Zäsur, die die Gegenwartsstarre unterbricht, wahrzunehmen und als solche $\mathrm{zu}$ erkennen,

50 Ebd.

51 Ebd., S. 129.

52 Ebd., S. 146. 
selbst als sie direkt vor seinen Augen geschieht. Gegen Ende seines Aufenthalts in Athen erlebt er an mehreren Tagen Demonstrationen und zuletzt gewaltsame Aufstände im Stadtteil Exarchia, der tatsächlich für seine teilweise heftigen Straßenkämpfe zwischen Autonomen und der Polizei bekannt ist. Als Arnold mit Odile und Esther das erste Mal in Exarchia ist, sieht er durch das Straßenfenster eines Restaurants Demonstrant*innen, die vor der Polizei fliehen, während Esther die Handlung eines Films beschreibt. Die Diskrepanz zwischen Esthers fiktionaler Erzählung und dem realen Geschehen, das durch die Scheibe des Fensters wahrgenommen wird, lösen eine ähnliche Dissoziation aus, wie die Gleichzeitigkeit des Verschiedenen, das über digitale Medien vermittelt wird:

Zwei Männer rannten zu schnell, in echter Angst vorbei. Ein paar Sekunden später folgte eine Menge schwarzgekleideter Frauen und Männer mit Motorradhelmen. Die Verfolgten versteckten sich in einem Café. Aus dem Nichts tauchten Eisenstangen auf. Die schwarzgekleideten Frauen und Männer schlugen das Schaufenster des Cafés ein. Arnold blickte eine unbeteiligte Frau mit Einkäufen an. Sie zuckte mit den Achseln. Die schwarze Funktions kleidung, die die meisten trugen, war von ein und derselben Trekking-Marke. Wut, Aktivismus, Interesse für die Weltlage, dachte Arnold, auch alles potentielle Konsumzapfhähne. ${ }^{53}$

Die Gleichgültigkeit, mit der Arnold die politischen Kämpfe auf der Straße im Zusammenspiel mit kapitalistischen Interessen betrachtet, ist ein Hinweis darauf, dass in der Konsumlogik des Kapitalismus auch Aufstände und Revolutionen letztlich konsumierbare Ereignisse sind. In einem Reiseführer wird der Stadtteil als „von sogenannten Anarchisten regiert“ ${ }^{* 54}$ beworben, Arnold empfindet die Umgebung als eine „Kulisse für linksalternative Tourismuserfahrung“ und beobachtet eine „alternative Krisen-Stadtführung“, bei der die Touristinnen aufgefordert werden, mit den Straßenhändler^innen um Second-Hand-Kleidung zu feilschen..$^{55}$ Die ,angenehme folkloristische Abwechslung “,${ }^{56}$ als die Arnold die Zurschaustellung politischer Kämpfe empfindet, verweist auf eine Logik des Kapitalismus, der er sich selbst nicht entziehen kann. Ähnlich wie der Terroranschlag in Paris lösen auch die teilweise gewalttätigen Demonstrationen bei Arnold keine entsprechende Reaktion aus. Beide Ereignisse erscheinen auf die gleiche Weise als konsumierbare Gegenwart.

Selbst als er sich einige Tage später selbst mitten in gewalttätigen Ausschreitungen wiederfindet, empfindet er keine entsprechende emotionale Reaktion zu den Ereignissen, die er weiterhin eher als medial vermitteltes Spektakel

53 Ebd., S. 123.

54 Ebd., S. 118.

55 Ebd.

56 Ebd. 
denn als die potenzielle und von ihm erhoffte Zäsur der Gegenwartserfahrung wahrnimmt: „Arnold stellte sich zu einer Gruppe Touristen mit China-Nudeln in der Hand, die sich fragten, wo der lustige Junkie mit der Fuck-the-Police-Flagge war, mit dem sie sich eben noch unterhalten hatten. Irgendjemand hatte den Redemption Song angemacht. “57 Obwohl Arnold sich in einer durchaus gefährlichen Situation befindet, erscheinen die Gewalt, das Feuer, die fliegenden Steine und Flaschen um ihn herum lediglich als Teil eines Spektakels für Touris$t^{\star}$ innen, die teilweise auch selbst daran teilnehmen. Als sich die Situation um Arnold beruhigt, stellt er wie nach dem Ende eines Konzerts oder einer Veranstaltung fest: „Das scheint es für heute gewesen zu sein. “58 In dieser indifferenten Haltung Arnolds gegenüber den Straßenkämpfen, dem aktiven politischen Kampf, der sich in seiner direkten Umgebung abspielt, ist eine Unfähigkeit erkennbar, zwischen der physischen Realität der Gewalt und ihrer medialen Repräsentation zu unterscheiden. Genauso wie Arnold das Attentat in Paris und weitere aktuelle Ereignisse mehr oder weniger emotionslos auf seinem Laptop oder seinem iPhone beobachtet, registriert er auch die Gewalt um ihn herum als medial vermitteltes Schauspiel. Die Szene während der Demonstration und der Straßenkämpfe wirkt wie ein Verweis auf einen Moment wenige Tage zuvor in Paris, als er noch lediglich Videos von Demonstrationen angeschaut hatte:

Er schaute sich ein paar Videos der Unruhen von 2005 an, und er dachte, wie zufrieden ihn die Aufnahmen plündernder Menschen damals gestimmt hatten. Die Bilder waren ihm wichtig oder neu vorgekommen, weil er dieses schaukelnde, rauschende SchwarzWeiß-Bild einer Kameradrohne nie zuvor gesehen hatte. Sein jugendliches Ich war davon überzeugt gewesen, dem Beginn von etwas sehr Großem beizuwohnen, zum Beispiel dem Ende des Kapitalismus. Arnold wusste damals noch nicht, dass alles verebbt, alle Aufstände und jedes Gefühl. ${ }^{59}$

Rückblickend scheint dieser Moment ein proleptischer Verweis auf Arnolds Wahrnehmung der tatsächlichen Proteste wenige Tage später in Athen zu sein. Hatte er über zehn Jahre zuvor Unruhen als bedeutend wahrgenommen, nicht zuletzt, weil die Imperfektion der Videoaufnahmen Relevanz und Authentizität suggeriert hatte, sieht er sich nun - selbst als teilweise partizipativer Teil von politischen Unruhen - nicht in der Lage ihre Bedeutung zu erkennen oder sich emotional in Beziehung zu ihr zu setzen. Was hier angedeutet scheint, ist eine Unfähigkeit die Realität als solche wahrzunehmen, weil sie bereits durch mediale Vermittlung mehrfach in ähnlicher Form erlebt wurde. Die Hyperrealität

57 Ebd., S. 154.

58 Ebd., S. 157.

59 Ebd., S. 17. 
medial vermittelter Ereignisse löst bei Arnold eine Ent-Täuschung im Wortsinn aus: Die Realität wirkt im Vergleich zu ihrem Simulacrum nicht realistischer. Erst als er die sich ihm darbietende Szenerie mit dem iPhone fotografieren will, wird das reale Geschehen der Proteste wieder ästhetisiert: „In einer dunklen Gasse nahm er sein iPhone und schoss ein Foto: im Vordergrund eine geschmolzene Plastikmülltonne vor Athener Apartmenthäusern, im Hintergrund Sirenenlicht und sich neu zusammenrottende Polizisten."60 Das digitale Festhalten der Realität erzeugt in dem Moment die „simulierende Dimension der Hyperrealität“61 im Sinne Baudrillards. Dass Arnold jedoch bevor er das Foto tatsächlich machen kann, das iPhone von einem Polizisten weggerissen und er selbst in Gewahrsam genommen wird, ist nur folgerichtig in der Konsequenz der Zeitwahrnehmung, die erneut am Erleben einer Zäsur scheitert.

\section{Die Gegenwart als zeitlose Schwellenphase}

Das Ende des Romans ist geprägt von einem Gefühl von Zeitlosigkeit, die Arnold zunächst in Folge des verlorenen iPhones überkommt. Mit dem Verlust der permanenten Verbindung zu einer medial vermittelten Gegenwart setzt für Arnold eine Form der Zeitwahrnehmung ein, die zwar nicht mehr geprägt ist von einer starren Gegenwart, die jedoch auch nicht in eine sinnhafte Zukunft reicht. Nicht in die Möglichkeit einer Zeit- oder Gegenwartserfassung in Form digitaler Medien eingebunden zu sein, erzeugt hier Zeitlosigkeit. Als er, nachdem die Polizei ihn freigelassen hat, auf einer Anhöhe über die Stadt blickt, denkt er „,an die simple Ordnung des Universums zu seiner Anfangszeit. An den Beginn der Zeit. Und Arnold wurde klar, dass er ein Ausdruck von Komplexität war, den das Universum erreicht hatte. Ihm wurde klar, dass die silberne Kette, die Odile und er sich vor weniger als einem Jahr online bestellt hatten, von einer Supernova vor Milliarden Jahren geschmiedet worden war. “62 Der Zeitraum, der hier aufgerufen wird, ist weniger ein Blick in eine konkrete Vergangenheit, sondern vielmehr auf eine temporal losgelöste Ebene, in der eine Gegenwart, wie sie Arnold bisher erfahren hat, keine Rolle mehr spielt: „Er wusste nicht, wie lange er dort stand.“63

60 Ebd., S. 157.

61 Jean Baudrillard: Der symbolische Tausch und der Tod. München 1982, S. 116.

62 Goldhorn: Park, S. 162.

63 Ebd. 
Als er sich schließlich am Flughafen von Athen wieder von Esther und Odile verabschiedet, besteht keinerlei Verbindung mehr zu einer digital vermittelten Gegenwart; während Arnold über kein Smartphone mehr verfügt, haben die anderen beiden keine Netzverbindung. Beinahe überzogen symbolisch tritt Arnold damit in ein Umfeld ein, das außerhalb von Zeit stattfindet. In Folge der Aufstände der Nacht zuvor sind sämtliche Strom- und Kommunikationsnetze der Stadt zusammengebrochen und Arnold ist gezwungen in einem Hotel zu übernachten, das aber selbst über keine Elektrizität oder einen Internetzugang verfügt. Während er dort darauf wartet, in sein Zimmer gehen zu können, stößt er in einem Kunstkatalog auf das Gemälde Abend über Potsdam von Lotte Laserstein aus dem Jahr 1930, in dessen zentraler weiblicher Figur er Odile zu erkennen meint. Die Bedeutung, die damit am Ende des Romans auf dieses Gemälde gelegt wird, rückt die bisherige Gegenwartserfahrung Arnolds in einen historischen und politischen Kontext.

Lasersteins Darstellung eines mondänen Abends vor dem Hintergrund Potsdams ist, so Kristin Schroeder in einer ausführlichen Analyse des Gemäldes, die Darstellung des „political hangover in the aftermath of Weimar's decadent years“. ${ }^{64}$ Laserstein zeige einen Moment des Übergangs, indem sie einerseits durch Anklänge an Vermeer und Leonardo da Vinci auf die Kunsthistorie verweise, andererseits aber einen konkreten Moment ihrer Gegenwart abbilde. Die Positionierung junger Menschen aus der gegenwartsbegeisterten Metropole Berlin in einem historischen Vorstadtumfeld deutet zudem die Spannungen zwischen der Gegenwart der Weimarer Republik und der bereits dräuenden gesellschaftlichen und politischen Umwälzungen und Verbrechen in der nahen Zukunft der dreißiger Jahre an. ${ }^{65}$ Die Zeit scheint in dem Gemälde ebenso stillzustehen, wie die Gegenwart, in der Arnold sich befindet. Die abgebildeten Personen in Abend über Potsdam sind eben nicht in der pulsierenden Großstadt, sondern im ländlichen und ruhigen Potsdam. Der dargestellte Moment wird zusätzlich eingefroren, darauf weist Schroeder hin, da die Flüssigkeit, die die junge Frau rechts im Bild in ein Glas gießen will, im Moment, da sie über den Rand des Krugs fließen will, im Stillstand des Gemäldes eingefangen ist: „Laserstein decelerates time and delays the completion of the event, thwarting progress indefinitely. As time lags and the figures linger, the apparent solidity of the scene begins to crumble, offering a deceptively incohesive world in suspension. " ${ }^{\text {} 66}$ Das

64 Kristin Schroeder: „An Ambivalent Elegy: Lotte Laserstein’s Evening Over Potsdam (1930)“. In: Art History. 42/4 (2019), S. 808-826, hier S. 812.

65 Vgl. ebd.

66 Ebd. 
Gemälde spiegelt daher in seiner rückblickenden Symbolhaftigkeit ${ }^{67}$ im Kontext des Romans in gewisser Weise die Situation, in der sich Europa am Übergang zum kommenden Jahrzehnt, den zwanziger Jahren des 21. Jahrhunderts, befindet. Besonders symbolisch mutet da das Ende des Romans in Athen an. Athen, als die Hauptstadt Griechenlands, ist mit der Finanzkrise von 2010 beinahe zum Symbol für die ersten große Risse im europäischen Selbstbild dieses Jahrhunderts geworden und steht durch die menschenunwürdigen Camps für Geflüchtete auf Lesbos gleichzeitig für die aktuelle Identitäts- und Humanitätskrise der EU am Übergang zu den 2020er Jahren. Arnold jedoch sieht in der zentralen Figur des Gemäldes lediglich Odile, die er in dem Moment als „die erste Bewohnerin seiner Zukunft“68 zu erkennen meint. Er steht damit in seinem Scheitern an Erkenntnis auch in der Tradition eines berühmten Protagonisten deutscher Popliteratur.

Von ersten Rezensionen wurde Park in den Bereich der Popliteratur gerückt und an seine dementsprechenden Vorgänger angebunden. ${ }^{69}$ Bereits Christian Krachts namenloser Protagonist in Faserland reiste 1995 auf der Suche nach etwas, das sich nie genau manifestierte, und zeigte sich als junger Mann ohne Halt und Bewusstsein für die eigene Position in der unmittelbaren Gegenwart. Es erscheint daher beinahe zu naheliegend Arnold als die für das Jahr 2020 aktualisierte Version dieser übermächtigen Repräsentationsfigur der deutschen Popliteratur zu verstehen. Goldhorn strukturiert aber gerade das Ende seines Romans mit Nachdruck auf diesen Vergleich hin. Krachts Erzähler sucht im letzten Teil von Faserland vergeblich auf einem Züricher Friedhof nach dem Grab von Thomas Mann und verschwindet danach ohne Hinweis auf eine Zukunft in der Mitte des Züricher Sees. Die ergebnislose Suche nach der Ruhestätte des deutschen Großschriftstellers Mann wurde auch als die symbolische, scheiternde Suche nach einer deutschen Identität - gemäß dem Mann-Zitat „Where I am, there is Germany“ - interpretiert. ${ }^{70}$ Parallel dazu ist Arnold am Ende von Park unfähig in dem Gemälde Abend über Potsdam etwas anderes zu erkennen als seine Freundin und zeitweise Geliebte Odile, mit der er eine Zukunft imagi-

67 Das Städelmuseum beschreibt das Gemälde „[a]us unserer heutigen Perspektive [...] geradezu als Symbol einer Zeitenwende.“ Alexander Eiling: „Zeitenwende. Lasersteins Abend über Potsdam“ In: Städelblog. Städel Museum Frankfurt a.M.. https://blog.staedelmuseum.de/lottelaserstein-abend-ueber-potsdam/, 2019 [zuletzt eingesehen am 7.6.2021].

68 Goldhorn: Park, S. 173.

69 Siehe dazu: Carla Kaspari: „Ich kann Bedürfnisse viel besser online ausdrücken“. In: Die Zeit 47/2020 (12.11.2020), S. 56.

70 Vgl. Michael Peter Hehl: „Kracht, Christian: Faserland. Roman“. In: Heribert Tommek u.a (Hg.): Wendejahr 1995. Transformationen der deutschsprachigen Gegenwartsliteratur. Berlin/ Boston 2015, S. 426-437, hier S. 433. 
niert, die angesichts der Trennung und des dargestellten Verhältnisses als äußerst unwahrscheinlich erscheint. In der Konsequenz dieser Unfähigkeit den entscheidenden Hinweis auf seine Suche nach Zukunft zu erkennen, verschwindet Arnold ähnlich wie der Faserland-Erzähler in der Zeitlosigkeit eines Hotelzimmers ohne Strom und Internetverbindung.

Als er schließlich mit seinem Laptop auf dem Hotelbett sitzt und alle Programme außer dem TextEdit-Programm geschlossen hat, markiert nun der schwächer werdende Akku die vergehende Zeit:

Bei $71 \%$ hatte er drei Gedichte geschrieben.

Bei $56 \%$ hatte er sieben Gedichte geschrieben.

Bei $23 \%$ hatte er mit einem Website-Generator eine Internetseite gebaut.

Bei $22 \%$ schrieb er das Gedicht seines Traumes aus Paris neu. ${ }^{71}$

Damit wird schließlich zum Ende des Romans der Verlauf von Zeit in der Wahrnehmung Arnolds konkret an ein digitales Gerät gebunden. Als der Akku leer ist und Arnold ihn aufgrund fehlender Elektrizität auch nicht mehr laden kann, scheint auch die Zeit um Arnold herum stillzustehen: „Arnold bewegte sich nicht. “72 So lautet der letzte Satz und bringt damit auch die Zeit für Arnold zu einem Ende. Entscheidend für die Wahrnehmung und Darstellung von Zeit innerhalb von Park ist jedoch, dass Arnold vor diesem letztendlichen Stillstand aus dem Fenster Menschen sieht, die den Flughafen stürmen. Es wird also eine Zukunft geben, vielleicht sogar eine grundlegend neue Zukunft in Form der Zäsur, die Arnold herbeigesehnt hat. Die Zeit bleibt nur in Arnolds Wahrnehmung stehen. Damit steht am Ende des Romans eine Trennung zwischen der starren Gegenwartswahrnehmung von Arnold in einem digitalen Umfeld und dem tatsächlichen Verlauf der Zeit, der unabhängig von dieser Wahrnehmung geschieht und damit auch eine Zukunft enthält.

\section{Fazit - Nicht neu, aber gegenwärtig}

Heinrich Heine und Rainer Maria Rilke leben und schreiben im 19. und zu Beginn des 20. Jahrhunderts beide in einer Zeit des rasanten technischen Fortschritts. In der Lebenszeit von Heine in der ersten Hälfte des 19. Jahrhunderts wird nicht nur die dampfbetriebene Eisenbahn erfunden, die bald weit voneinander entfernte Orte verbindet, sondern auch das erste elektrische Licht entwi-

71 Goldhorn: Park, S. 177.

72 Ebd., S. 179. 
ckelt, sowie die Telegrafie im größeren Umfang ausgebaut. In den ersten Lebensjahrzehnten von Rilke wird das Telefon patentiert, elektrisches Licht wird nun kommerziell verfüg- und dadurch verbreitet nutzbar, das Auto wird erfunden und die Brüder Lumière entwickeln den Kinematographen. Gleichzeitig und dadurch bedingt und beschleunigt entwickelt sich die Großstadt als Lebensraum und gesellschaftliches Konzept und quasi als eigene Form des $\mathrm{Zu}$ sammenlebens. ${ }^{73}$ In den zu Anfang zitierten Passagen aus Heines Pariser Zeitungsartikeln und aus Rilkes fiktionalisierten Aufzeichnungen der eigenen Großstadterfahrungen scheint etwas hindurch, was zumindest bei Rilke durchaus eine zentrale Rolle spielt, was sich aber insbesondere durch den gesamten Roman Park zieht: Eine perzeptive Überforderung aufgrund der technischen Entwicklung und dadurch veränderter Wahrnehmungsverhältnisse. Und sowohl bei Heine und Rilke als auch bei Goldhorns Protagonisten Arnold hängt diese Überforderung zusammen mit einer Verschiebung des Zeit-Raum-Verhältnisses. Während sich diese Auseinandersetzung mit temporalen und spatialen Kategorien in den Texten von Heine und Rilke lediglich am Rande zeigt, steht sie bei Goldhorn im Zentrum der Narration.

Die Rückgriffe auf die Zeit eines rasanten technischen Fortschritts im 19. Jahrhundert und damit einhergehende Überforderungen, die unter anderem Georg Simmel 1903 als eine „Steigerung des Nervenlebens, die aus dem raschen und ununterbrochenen Wechsel äußerer und innerer Eindrücke hervorgeht“ ${ }^{74}$ beschreibt, zeigen jedoch, dass die perzeptive Überforderung angesichts ständig wechselnder und neuer Sinneseindrücke keine grundlegend neue Erfahrung des digitalisierten 21. Jahrhunderts ist. Gerade die Frage, die Schivelbusch aufwirft, ob die „Eisenbahn, der Beschleuniger der industriellen Revolution, und der Computer verschiedene Punkte auf derselben Kurve der Entwicklung von Maschinen einnehmen “ ${ }^{75}$ ist ein Hinweis darauf, dass Erfahrungen einer Überforderung aufgrund technischer Entwicklung keine genuine Erfahrung des frühen 21. Jahrhunderts sind. Schivelbusch stellt am Beispiel von Heines Wahrnehmung der brandenden Nordsee vor seiner Haustür in Paris fest, dass die beiden Orte Paris und die Nordseeküste - „ihr altes Hier und Jetzt" ${ }^{\text {“76 }}$ verlieren. Diese Fähigkeit Orte, die weit voneinander entfernt sind, medial zu vereinen und dadurch im ersten Moment eine Überforderung zu erzeugen, haben nicht nur die Eisenbahn,

73 Siehe dazu auch Georg Simmel: „Die Großstädte und das Geistesleben“. In: Ders.: Gesamtausgabe. Aufsätze und Abhandlungen 1901-1908. Hg. v. Otthein Rammstedt, Bd. 7/I, Frankfurt a.M. 2017, S. 116-131.

74 Ebd., S. 116.

75 Schivelbusch: „Weltmaschinen“, S. I.

76 Schivelbusch: Geschichte der Eisenbahnreise, S. 39. 
sondern in größerer Intensität und anderer Form auch der Computer und das Internet. Die Vernichtung von Zeit und Raum, die Schivelbusch konstatiert, und die dadurch hervorgerufene Überforderung erfährt auch Arnold in Park. Simmels Beschreibung einer solchen Überforderung in „Die Großstädte und das Geistesleben“ lässt sich beinahe ohne Veränderung auf Arnolds Gegenwartswahrnehmung in den Großstädten des 21. Jahrhunderts mit permanentem Internetzugang übertragen:

Der Mensch ist ein Unterschiedswesen, d. h. sein Bewußtsein wird durch den Unterschied des augenblicklichen Eindrucks gegen den vorhergehenden angeregt; beharrende Eindrücke, Geringfügigkeit ihrer Differenzen, gewohnte Regelmäßigkeit ihres Ablaufs und ihrer Gegensätze verbrauchen sozusagen weniger Bewußtsein, als die rasche Zusammendrängung wechselnder Bilder, der schroffe Abstand innerhalb dessen, was man mit einem Blick umfaßt, die Unerwartetheit sich aufdrängender Impressionen. ${ }^{77}$

Zwar lässt sich im Zuge des technischen Fortschritts, der Digitalisierung und der weiter beschleunigten Informations- und Kommunikationsinfrastruktur eine Forcierung und Zuspitzung dieser Entwicklung über den Verlauf des 20. und die ersten Jahrzehnte des 21. Jahrhunderts nicht leugnen, das ändert jedoch nicht die prinzipielle Feststellung, dass die Diagnose einer Überforderung nicht grundsätzlich neu ist. Sie wird lediglich an gegenwärtige technische und inzwischen auch digitale Umwelten angepasst. Park thematisiert so einen aktuellen theoretischen Diskurs über Zeitwahrnehmung und Gegenwart und bewegt sich dabei in dem Spektrum von Fortschrittsüberforderung und der Diagnose eines entropischen Stillstands durch Digitalisierung, das zahlreiche Debatten zu Gesellschaft und Digitalisierung der letzten Jahrzehnte geprägt hat. Mit Blick auf die Zeit der Industrialisierung lassen sich diese Erfahrungen auch an Diskurse des 19. Jahrhunderts anschließen.

Marius Goldhorns Park ist damit in gewisser Weise ein Zeitroman für das 21. Jahrhundert im umgekehrten Sinne. Er bildet einen gegenwärtigen gesellschaftlichen Zustand im Europa des frühen 21. Jahrhunderts durch die Perspektive einer einzelnen Person anhand aktueller Gegenwartsdiskurse ab. Anders als die Zeitromane des 19. und frühen 20. Jahrhunderts stellt er damit nicht durch verschiedene Repräsentationsfiguren ein gesellschaftliches Panorama dar, sondern geht den umgekehrten Weg, den einer Perspektivenverengung. Er ist zudem ein Zeitroman in dem Sinne, dass Zeit als Diskursthema und als entscheidender Parameter im Zentrum dieser Auseinandersetzung mit Gegenwart stehen. Zeit ist dabei in der personal eingeschränkten Perspektive der Narration keine unabhängige Größe, sondern grundlegend der Wahrnehmung des Protag-

77 Simmel: „Die Großstädte und das Geistesleben“, S. $116 \mathrm{f}$. 
onisten unterworfen. Im Fokus dieser Zeitwahrnehmung steht der Einfluss von Medien. Hervorzuheben ist dabei vor allem, wie deutlich sich Park an Thesen wie der des Present Shock von Rushkoff anlehnt. Gleichzeitig schließt der Roman an die genannten Theorien von Großklaus und Meyrowitz an und verbindet die permanente medial vermittelte Gegenwart im Zuge der Digitalisierung mit der Hyperrealitätstheorie von Baudrillard. Der Roman verengt damit seine Gegenwartsdiagnose auf eine Reihe medien- und zeittheoretischer Diskurse.

Gleichzeitig ist die These eines präsentischen, gesellschaftlichen Stillstands in Form einer Fokussierung auf die Gegenwart nicht zuletzt aufgrund von umfassender Digitalisierung, wie sie Rushkoff aufstellt und Goldhorn literarisch umsetzt, insofern in Frage zu stellen, als sie die zukunftsorientierten Nutzungsund Partizipationsmöglichkeiten des Internets und der Digitalisierung ignorieren muss, um ihre Aussage untermauern zu können. Politische und gesellschaftliche Bewegungen wie Fridays For Future oder Black Lives Matter, ebenso wie SocialMedia-basierte Aktionen wie \#metoo haben in den letzten Jahren gezeigt, wie gerade der technische und digitale Fortschritt und damit einhergehende Kommunikationsstrukturen dafür genutzt werden, Zukunftsperspektiven aufzuzeigen, statt gegenwärtige Zustände der Gesellschaft zu akzeptieren.

Park verbleibt somit in seiner zeitdiagnostischen Aussage in einem engen Bereich eines kulturpessimistischen Theoriediskurses, der in Teilen bereits im ausgehenden 19. Jahrhundert geführt wurde und der zu Beginn des 21. Jahrhunderts lediglich einen Teil des digitalen Fortschritts erfasst und seinen Blick nicht auf einen zukunftsorientierten Umgang damit richtet. Zwar steht am Ende des Romans durch das Gemälde von Laserstein auf Seiten der Erzählinstanz der Ausdruck eines Bewusstseins für eine Schwellenzeit, da diese jedoch von Arnold nicht erkannt wird, bleibt am Ende der Stillstand. 